\title{
The application of corticosteroids in COVID-19: A two-edged sword
}

\author{
Na Li ${ }^{1}$, Zhijun Jie ${ }^{1,2}$ \\ 'Department of Pulmonary and Critical Care Medicine, Shanghai Fifth People's Hospital, Fudan University, \\ Shanghai, China; \\ ${ }^{2}$ Center of Community-Based Health Research, Fudan University, Shanghai, China
}

\section{ABSTRACT}

COVID-19 has become a global pandemic and requires the whole world to respond together. There is no specific antiviral treatment recommended at present for COVID-19. The patients must receive the supportive care to help relieve the symptoms and ensure appropriate infection control. Whether or not to use corticosteroids clinically caused controversy. This article has summarized previous researches about the using of corticosteroids in other viral pneumonia, related clinical data in COVID-19, and recommendations in Chinese guideline.

Key words: COVID-19, corticosteroid, cytokine storm

\section{INTRODUCTION}

Since December 2019, a new strain of the coronavirus called severe acute respiratory syndrome coronavirus 2 (SARS-CoV-2) has appeared in Wuhan, Hubei Province, China. The disease caused by SARS-CoV-2 was officially named "COVID-19" by the World Health Organization on February 11, 2020. COVID-19 has become a pandemic, which spread across the world including more than 211 countries or regions and infected $1,914,916$ patients globally (83,747 in China) by April 15, 2020. ${ }^{[1]}$ SARS-CoV-2, which has a typical genome structure of $\mathrm{CoV}$, belongs to the cluster of betacoronaviruses; this new virus and SARS-CoV have approximate $70 \%$ similarity in gene sequence. ${ }^{[2,3]}$ There are some differences in lethality and infectivity between SARS-CoV-2 and other coronaviruses. Early data showed that compared with SARS-CoV and Middle East respiratory syndrome coronavirus (MERS-CoV), which also caused severe respiratory syndrome in humans, the mortality of COVID-19 was lower but the contagion was very higher. ${ }^{[4-6]}$ In early stage of the outbreak of COVID-19, China has adopted a series of measures to block the explosive spread of this virus. Although some antiviral drugs, convalescent plasma transfusion, and chloroquine phosphate have been proven to have partial efficacy in the treatment of COVID-19, there is still no specific antiviral treatment recommended at present for COVID-19. Whether or not to use corticosteroids clinically caused controversy. This article has summarized previous researches about the using of corticosteroids in other viral pneumonia, related clinical data in COVID-19, and recommendations in Chinese guideline.

\section{COVID-19 AND THE CYTOKINE STORM}

The cytokine storm is an important cause of acute respiratory distress and multiple organ failure, which seriously threatens patients' life.

Zhou et al..$^{[7]}$ first described the risk factors for the mortality of 53 nonsurvivors and 118 survivors with COVID-19 in a retrospective, multicenter, cohort study. The result showed that higher Sequential Organ Failure Assessment (SOFA) score (5.65, 2.61-12.23; $P<0.0001)$ was associated with increased odds of death. Sepsis was the most common complication in all patients, followed by respiratory failure, acute respiratory distress syndrome (ARDS), or heart failure. The frequency of these complications and levels of IL-6 were all 
higher in nonsurvivors compared with survivors. These severe symptoms explained the difficulty in treatment and led us to wonder what role the cytokine storm has played in patients with COVID-19.

Qin et al. ${ }^{[8]}$ analyzed the clinical data of 452 patients with COVID-19 in a retrospective single-center study and found most of them tended to have elevated inflammatory cytokines. Plasma levels of IL-6, IL-8, IL-10, and TNF- $\alpha$ in patients with severe infection were significantly higher than those in patients with nonsevere infection, similar to the results in SARS and MERS. ${ }^{[9,10]}$ In a report, the average level of IL-6 in 99 patients was much higher than the upper limit of the normal range. However, the author did not analyze the relationship between increased inflammatory factors and clinical outcomes. ${ }^{[1]}$ Liu et al. ${ }^{[12]}$ described the dynamic changes in inflammatory cytokines in patients with COVID-19. The levels of IL-6 and IL-10 showed a continuous increase in patients with severe infection. The levels of cytokines in patients with severe infection who survived gradually returned to the levels that were comparable with those of mild cases. The results concluded that the progression of COVID-19 could be predicted from cytokines. ${ }^{[13]}$

As changes in inflammatory cytokines were related to the severity of COVID-19, it should be beneficial for critical patients to suppress the cytokine storm. Corticosteroids could achieve the purpose of suppressing lung inflammation, but they can also restrain the immune response. This contradictory aspect put the application of corticosteroids in an awkward position, especially the results of corticosteroid therapy in previous SARS, MERS, and influenza seemed to be uncertain.

\section{CORTICOSTEROID THERAPY IN SARS, MERS AND INFLUENZA}

Corticosteroids have unique advantages in inhibiting inflammatory responses and have been previously used in the treatment of SARS, MERS, and influenza. However, its uncertain efficacy and adverse reactions make doctors hesitate to use corticosteroids in the treatment of COVID-19. Here we reviewed the application of corticosteroids in the previous infectious diseases, hoping to provide some references for the use of corticosteroid in COVID-19.

Auyeung et al. ${ }^{[14]}$ performed a retrospective cohort study on 78 adult patients with SARS in Hong Kong. Sixty-six of 78 (84.6\%) patients accepted different doses of corticosteroids, and the result showed that corticosteroid treatment was associated with a 20.7-fold increase in the risk of either ICU admission or mortality. But only advanced age and high
LDH levels were used to eliminate confounding effect by multivariate adjustment; the results of this study could be biased by many unknown confounding factors. Stockman et al. ${ }^{[15]}$ conducted a systematic review and comprehensive summary of the treatments used for patients infected with SARS. The possible harm, such as delayed virus clearance, onset of diabetes, avascular necrosis, and osteoporosis, were reported in patients with SARS treated with corticosteroid. One small RCT showed that low-dose rather than highdose corticosteroids may be beneficial for the improvement of ARDS. Zhao et al. ${ }^{[16]}$ aimed to examine the effects of steroid therapy on osteonecrosis in recovered patients with SARS in a meta-analysis. They observed a nonlinear relationship between osteonecrosis and the cumulative dose and treatment duration of steroids. Their further analysis indicated that the risk of osteonecrosis remained relatively low when the total dose of steroids (methylprednisolone) was $\leq 5 \mathrm{~g}$ and the treatment duration was $<30$ days. Chen et al. ${ }^{[17]}$ reviewed 401 SARS cases confirmed by virology in Guangzhou, Guangdong Province of China, to evaluate the efficacy and safety of steroid therapy. Their study revealed that proper corticosteroid treatment resulted in lower mortality and shorter hospitalization stay in patients with critical SARS with an oxygenation index (OI) of $<300 \mathrm{~mm}$ $\mathrm{Hg}$, and it was not associated with significant secondary lower respiratory infection and other complications.

Arabi et al. ${ }^{[18]}$ used marginal structural models to adjust for baseline and time-varying confounders to investigate the relationship between systemic corticosteroid treatment and 90-day mortality in patients with MERS admitted to ICU. They found that the dose and time of initiation of corticosteroid therapy had no impact on 90-day mortality. But early initiation of corticosteroid therapy was associated with delayed MERS coronavirus RNA clearance. Arabi et al. also mentioned that persistent positivity of MERSCoV RNA did not necessarily mean persistent shedding of live virus. Alfaraj et al. ${ }^{[19]}$ performed a cohort study and examined clinical predictors of the mortality of MERS infection. This study found that advanced age, increased WBC and neutrophil count, lower serum albumin level, use of CRRT, and use of corticosteroid were associated with increased mortality. The odd ratio for mortality was second highest for the use of corticosteroid. Confuse factor disturbance in this study was not removed, and they did not know whether all factors contributed to mortality.

A systematic review and meta-analysis were conducted to provide evidence regarding corticosteroids in the treatment of influenza. The result of this meta-analysis suggested that the use of corticosteroids increased the risk of mortality and hospital-acquired infection. Patients in this study mainly received high doses of corticosteroids, and the evidence was of low quality with potential 
confounding. ${ }^{[20]} \mathrm{Li}$ et al. ${ }^{\left[{ }^{[1]}\right.}$ described the effect of different doses of adjuvant corticosteroid therapy on 30- or 60-day mortality of patients with influenza A (H1N1) pdm09 viral pneumonia through a retrospective analysis. A total of 2141 hospitalized adolescent and adult patients were included in the study. The results of stratified analysis based on the doses of corticosteroids showed that only treatment with low- to moderate-dose corticosteroid could reduce 30- and 60-day mortality of patients with severe infection with $\mathrm{PaO} 2 / \mathrm{FiO} 2<300 \mathrm{~mm} \mathrm{Hg}$. However, corticosteroids at any dose increased the 60-day mortality of patients with mild infection with $\mathrm{PaO} 2 / \mathrm{FiO} 2>300 \mathrm{~mm} \mathrm{Hg}$. Cao et al. ${ }^{[22]}$ screened patients aged $>14$ years with severe influenza A (H7N9) virus infections from April 2013 to March 2015 to determine the impact of corticosteroids. They found that high-dose corticosteroid therapy $(>150 \mathrm{mg} / \mathrm{d}$ methylprednisolone or equivalent) was associated with longer viral shedding and significantly increased both 30 and 60-day mortality.

On the whole, corticosteroid therapy seemed to be a double-edged sword in these infectious diseases. So corticosteroids, especially high-dose corticosteroids, should be used in COVID-19 more cautiously. Of course, it would be better if supported by high-quality randomized controlled trials when corticosteroids are used.

\section{RELATED CLINICAL DATA IN COVID-19}

As there is no strong clinical evidence that corticosteroids can benefit patients with COVID-19, and worrying about the possible harm, corticosteroids are not widely used in the treatment of COVID-19. For the above reasons, Clark Russell and colleagues concluded that COVID-19related lung injury or shock should not be treated with corticosteroids except for clinical trials. ${ }^{[23]}$ Although the Lancet published the world's first pathological results of a patient with COVID-19 on February 17, 2020. The patient's lungs showed diffuse alveolar injury and pulmonary hyaline membrane formation consistent with the performance of ARDS, suggesting that corticosteroids should be provided timely and appropriately. ${ }^{[24]}$ So far, whether the patients with COVID-19 could benefit from corticosteroids therapy is not clear. At this time, it is important to summarize the advantages and disadvantages of corticosteroid therapy in COVID-19.

On the negative side, Liu et al. ${ }^{[25]}$ reported the clinical characteristics and treatment of patients with COVID-19 with ARDS in a study available on the medRxiv preprint server. This study enrolled 109 patients in Wuhan, China, from January 2 to February 1, 2020. They concluded that patients with moderate or severe ARDS were more likely to receive corticosteroids, but the effects of corticosteroid treatment on the survival were not satisfactory. Similarly, Feng et al. ${ }^{[26]}$ found that more patients received corticosteroids in the critical and severe groups. Although corticosteroid therapy did not have any association with disease progression to death or mechanical ventilation, receiving no corticosteroids in the critical group was associated with good prognosis. Shang et al. ${ }^{[27}$ divided 416 patients with COVID-19 into three groups based on clinical outcomes: the common survivor (not severe/ critical) group, the severe/critical survivor group, and the death group. The comparison of corticosteroid therapy among different groups showed that corticosteroid therapy prolonged the hospitalization days for the patients in the common and severe/critical survivor groups. Patients in the two survivor groups who received corticosteroid treatment had lower lymphocyte counts and the median percentage of lymphocytes was reduced after corticosteroid therapy in the death group. It is better to analyze more cases in this retrospective observational study.

What are the positive effects of corticosteroids? The efficacy of corticosteroids for severe or fatal cases with COVID-19 in Wuhan was evaluated. The results showed that although low-dose corticosteroids might not improve ICU mortality, it suppressed the cytokine storm in the phase of ARDS and effectively improved the blood oxygen saturation. This result implied that corticosteroid therapy might provide valuable time for these patients in controlling infection and preventing secondary multiorgan damage and shock. An accurate conclusion could not be made because of the deficiency of sample size and the lack of matched control groups. ${ }^{[28]}$ There is a retrospective cohort study of 201 patients with COVID-19 whose median time from admission to progression to ARDS was only 2 days. Among the patients with ARDS, 23 of $50(46.0 \%)$ patients receiving methylprednisolone and 21 of $34(61.8 \%)$ patients who did not receive methylprednisolone died. These data suggested that the use of methylprednisolone appeared to reduce the risk of death in patients with ARDS (HR, 0.38; 95\% CI, 0.20-0.72). ${ }^{[2]}$ Zhou et al. ${ }^{[30]}$ analyzed the clinical data of 10 patients with COVID-19 and found that the lymphocyte count and OI of these patients with COVID-19 became more worrying after the treatment with a combination of low-dose methylprednisolone $(80 \mathrm{mg} / \mathrm{d})$ and immunoglobulin $(10$ $\mathrm{g} / \mathrm{d}$ ), and the pulmonary CT revealed that the lung lesions were significantly worse. However, the condition of these patients significantly improved after doctors increased doses of methylprednisolone and immunoglobulin to 160 and $20 \mathrm{~g} / \mathrm{d}$, respectively. So choosing the proper doses of corticosteroids is also a big challenge. This study was limited by small sample size and short-term follow-up of only 1 month. 
Although corticosteroids seemed to be beneficial for some patients with COVID-19, the sample sizes of these studies mentioned above were not large, and they were not randomized controlled experiments. Professor Cao also considered that it would be necessary to carry out the highquality trials in the future. ${ }^{[31]}$ Now it is still difficult to draw any firm conclusions. Following the guidance provided by experts is a good choice.

\section{RECOMMENDATIONS IN CHINESE GUIDELINE}

A report published by the China Medical Treatment Expert Group for COVID-19 concluded that 18.6\% of 1099 patients with COVID-19 received systemic corticosteroids, including 77 of 173 patients with severe infection or critical patients. ${ }^{[32]}$ Corticosteroids seemed to be empirically used by clinicians. There were different recommendations about the use of corticosteroids in guidelines for COVID-19.

On the basis of fully learning from the treatment experience of their counterparts at home and abroad, the Shanghai Clinical Treatment Expert Group for COVID-19 continued to optimize and refine the treatment plan for COVID-19 and formed an expert consensus, commonly known as "Shanghai Plan." The plan suggested that if there is a risk of becoming severe for patients with COVID-19, such as significant radiographic progress in pneumonia, $\mathrm{SaO} 2$ of patients $<93 \%$ in the resting state without oxygen therapy, or shortness of breath (breathing frequency $\geq 30$ times/minute), OI $\leq 300 \mathrm{~mm}$ $\mathrm{Hg}$, or facing the risk of intubation, low-dose shortcourse glucocorticoids are recommended generally for 3-6 days. ${ }^{[33]}$ Actually, early in the epidemic, the National Health Commission of China mentioned that glucocorticoids can be used for a short period of time based on the degree of dyspnea and chest radiographic progress. The recommended dose does not exceed 1-2 $\mathrm{mg} / \mathrm{kg} / \mathrm{d}^{\left[{ }^{[34]}\right.}$ Subsequently, multiple Chinese physicians developed an expert consensus as a supplement to this above guideline. The indications for glucocorticoid therapy are similar to those of the "Shanghai Plan," and it is better to follow these principles: (1) never use glucocorticoids to relieve the fever; (2) the dosage of glucocorticoids should be individualized according to different patients; and (3) if the situation of hypoxemia has already existed before suffering from COVID-19, be more cautious about indications of glucocorticoid therapy. In addition, the recommended time is not more than 7 days. ${ }^{[35]}$

With the accumulation of more experience and clinical data, therapeutic methods of corticosteroids will be continuously improved.

\section{CONCLUSIONS}

In general, there are still no very encouraging results about corticosteroids therapy for COVID-19. However, it is difficult to neglect that corticosteroids may have a potential role in the treatment of patients with COVID-19. Everything is inconclusive and we should remain optimistic. It is necessary to strictly abide by experts' advice and start or stop the use of corticosteroids in a flexible and timely manner when corticosteroids are used in the lack of highquality clinical trials that can provide more precise guidance for clinicians' work.

\section{Source of Foundation}

This study was supported by Key Research of Shanghai Fifth People's Hospital (2019WYZD03).

\section{Conflict of Interest}

None declared.

\section{REFERENCES}

1. WHO. Coronavirus disease 2019 (COVID-19) Situation Report-86. Available at: https://www.who.int/docs/default-source/coronaviruse/ situation-reports/20200415-sitrep-86-covid-19.pdf?sfvrsn=c615ea20_6. Access on April 1, 2020.

2. Chen Y, Liu Q, Guo D. Emerging coronaviruses: Genome structure, replication, and pathogenesis. J Med Virol 2020; 92: 418-23.

3. Hui DS, I AE, Madani TA, Ntoumi F, Kock R, Dar O, et al. The continuing 2019-nCoV epidemic threat of novel coronaviruses to global health-The latest 2019 novel coronavirus outbreak in Wuhan, China. Int J Infect Dis 2020; 91: 264-6.

4. Wang W, Tang J, Wei F. Updated understanding of the outbreak of 2019 novel coronavirus (2019-nCoV) in Wuhan, China. J Med Virol 2020; 92 : 441-7.

5. WHO. Summary of probable SARS cases with onset of illness from 1 November 2002 to 31 July 2003. Released on 26 September 2003. Available at: https://www.who.int/csr/sars/country/table2003_09_23/en/. Accessed on April 1, 2020.

6. WHO. Middle East Respiratory Syndrome Coronavirus (MERS-CoV). Available at: Accessed 25 January 2020. Available at http://www.who.int/ emergencies/mers-cov/en/. Accessed on April 1, 2020.

7. Zhou F, Yu T, Du R, Fan G, Liu Y, Liu Z, et al. Clinical course and risk factors for mortality of adult inpatients with COVID-19 in Wuhan, China: a retrospective cohort study. Lancet 20; 20395: 1054-62.

8. Qin C, Zhou L, Hu Z, Zhang S, Yang S, Tao Y, et al. Dysregulation of immune response in patients with COVID-19 in Wuhan, China. Clin Infect Dis 2020.pii: ciaa248.

9. Wong CK, Lam CW, Wu AK, Ip WK, Lee NL,Chan IH, et al. Plasma inflammatory cytokines and chemokines in severe acute respiratory syndrome. Clin Exp Immunol 2004; 136: 95-103.

10. Min CK, Cheon S, Ha NY, Sohn KM, Kim Y, Aigerim A, et al. Comparative and kinetic analysis of viral shedding and immunological responses in MERS patients representing a broad spectrum of disease severity. Sci Rep 2016; 6: 25359.

11. Chen N, Zhou M, Dong X, Qu J, Gong F, Han Y, et al. Epidemiological and clinical characteristics of 99 cases of 2019 novel coronavirus pneumonia in Wuhan, China: a descriptive study. Lancet 2020; 395: 507-13. 
12. Liu J, Li S, Liu J, Liang B, Wang X, Li W, et al. Longitudinal characteristics of lymphocyte responses and cytokine profiles in the peripheral blood of SARS-CoV-2 infected patients. EBioMedicine 2020; 55: 102763.

13. Wan S, Yi Q, Fan S, Lv J, Zhang X, Guo L, et al. Characteristics of lymphocyte subsets and cytokines in peripheral blood of 123 hospitalized patients with 2019 novel coronavirus pneumonia (NCP). medRxiv 2020:2020.02.10.20021832.

14. Auyeung TW, Lee JS, Lai WK, Choi CH, Lee HK, Lee JS, et al. The use of corticosteroid as treatment in SARS was associated with adverse outcomes: a retrospective cohort study. J Infect 2005; 51: 98-102.

15. Stockman LJ, Bellamy R, Garner P. SARS: systematic review of treatment effects. PLoS Med 2006; 3: e343.

16. Zhao R, Wang H, Wang X, Feng F. Steroid therapy and the risk of osteonecrosis in SARS patients: a dose-response meta-analysis. Osteoporos Int 2017; 28: 1027-34.

17. Chen RC, Tang XP, Tan SY, Liang BL, Wan ZY, Fang JQ, et al. Treatment of severe acute respiratory syndrome with glucosteroids: the Guangzhou experience. Chest 2006; 129: 1441-52.

18. Arabi YM, Mandourah Y, Al-Hameed F, Sindi AA, Almekhlafi GA, Hussein MA, et al. Corticosteroid Therapy for Critically Ill Patients with Middle East Respiratory Syndrome. Am J Respir Crit Care Med 2018; 197: 757-67.

19. Alfaraj SH, Al-Tawfiq JA, Assiri AY, Alzahrani NA, Alanazi AA, Memish ZA. Clinical predictors of mortality of Middle East Respiratory Syndrome Coronavirus (MERS-CoV) infection: A cohort study. Travel Med Infect Dis 2019; 29: 48-50.

20. Lansbury LE, Rodrigo C, Leonardi-Bee J, Nguyen-Van-Tam J, Shen LW. Corticosteroids as Adjunctive Therapy in the Treatment of Influenza: An Updated Cochrane Systematic Review and Meta-analysis. Crit Care Med 2020; 48: e98-106.

21. Li H, Yang SG, Gu L, Zhang Y, Yan XX, Liang ZA, et al. Effect of low-tomoderate-dose corticosteroids on mortality of hospitalized adolescents and adults with influenza $\mathrm{A}(\mathrm{H} 1 \mathrm{~N} 1)$ pdm09 viral pneumonia. Influenza Other Respir Viruses 2017; 11: 345-54.

22. Cao B, Gao H, Zhou B, Deng X, Hu C, Deng C, et al. Adjuvant Corticosteroid Treatment in Adults With Influenza A (H7N9) Viral Pneumonia. Crit Care Med 2016; 44: e318-28.

23. Russell CD, Millar JE, Baillie JK. Clinical evidence does not support corticosteroid treatment for 2019-nCoV lung injury. Lancet 2020; 395: 473-5.
24. Xu Z, Shi L, Wang Y, Zhang J, Huang L, Zhang C, et al. Pathological findings of COVID-19 associated with acute respiratory distress syndrome. Lancet Respir Med 2020; 8: 420-2.

25. Liu Y, Sun W, Li J, Chen L, Wang Y, Zhang L, et al. Clinical features and progression of acute respiratory distress syndrome in coronavirus disease 2019. medRxiv 2020:2020.02.17.20024166.

26. Feng Y, Ling Y, Bai T, Xie Y, Huang J, Li J, et al. COVID-19 with Different Severity: A Multi-center Study of Clinical Features. Am J Respir Crit Care Med 2020.

27. Shang J, Du R, Lu Q, Wu J, Xu S, Ke Z, et al. The Treatment and Outcomes of Patients with COVID-19 in Hubei, China: A Multi-Centered, Retrospective, Observational Study (2/26/2020). Available at SSRN: https:// ssrn.com/abstract=3546060. Accessed on April 1, 2020.

28. Zhou W, Liu Y, Tian D, Wang C, Wang S, Cheng J, et al. Potential benefits of precise corticosteroids therapy for severe $2019-\mathrm{nCoV}$ pneumonia. Signal Transduction and Targeted Therapy 2020; 5: 18.

29. Wu C, Chen X, Cai Y, Xia J, Zhou X, Xu S, et al. Risk Factors Associated With Acute Respiratory Distress Syndrome and Death in Patients With Coronavirus Disease 2019 Pneumonia in Wuhan, China. JAMA Intern Med 2020; Mar 13.

30. Zhou Z, Xie S, Zhang J, Zheng F, Jiang D, Li K, et al. Short-Term Moderate-Dose Corticosteroid Plus Immunoglobulin Effectively Reverses COVID-19 Patients Who Have Failed Low-Dose Therapy. Preprints 2020; 2020030065.

31. Shang L, Zhao J, Hu Y, Du R, Cao B. On the use of corticosteroids for 2019-nCoV pneumonia. Lancet 2020; 395: 683-4.

32. Guan W, Ni Z, Hu Y, Liang W, Ou C, He J, et al. Clinical Characteristics of Coronavirus Disease 2019 in China. N Engl J Med 2020; Feb 28.

33. Shanghai Clinical Treatment Expert Group for corona virus disease 2019. [Comprehensive treatment and management of corona virus disease 2019: expert consensus statement from Shanghai]. Chin J Infect Dis 2020; E016.

34. National Health Commission of the People's Republic of China. Guideline for the diagnosis and treatment of COVID-19 infections (version 1-7). 2020. http://www.nhc.gov.cn/yzygj/zcwj2/new_zcwj.shtml. Accessed on March 9, 2020.

35. Zhao JP, Hu Y, Du RH, Chen ZS, Jin Y, Zhou M, et al. [Expert consensus on the use of corticosteroid in patients with 2019-nCoV pneumonia]. Zhonghua Jie He He Hu Xi Za Zhi 2020; 43: 183-4.

How to cite this article: Li N, Jie Z. The application of corticosteroids in COVID-19: A two-edged sword. Transl Intern Med 2020; 8: 66-70 